\title{
Conditional Cuckoo Filters
}

\author{
Daniel Ting \\ Tableau Software \\ Seattle, Washington \\ dting@tableau.com
}

\author{
Rick Cole \\ Tableau Software \\ Palo Alto, California \\ ricole@tableau.com
}

\begin{abstract}
Bloom filters, cuckoofi lters, and other approximate set membership sketches have a wide range of applications. Oftentimes, expensive operations can be skipped if an item is not in a data set. These filters provide an inexpensive, memory efficient way to test if an item is in a set and avoid unnecessary operations. Existing sketches only allow membership testing for a single set. However, in some applications such as join processing, the relevant set is notfi xed and is determined by a set of predicates.

We propose the Conditional Cuckoo Filter, a simple modification of the cuckoofi lter that allows for set membership testing given predicates on a pre-computed sketch. Thisfi lter also introduces a novel chaining technique that enables cuckoofi lters to handle insertion of duplicate keys. We evaluate our methods on a join processing application and show that they significantly reduce the number of tuples that a join must process.
\end{abstract}

\section{KEYWORDS}

Bloomfi lter, cuckoofi lters, approximate set membership, semi-join

\section{ACM Reference Format:}

Daniel Ting and Rick Cole. 2021. Conditional Cuckoo Filters. In Proceedings of the 2021 International Conference on Management of Data (SIGMOD '21), fune 20-25, 2021, Virtual Event, China. ACM, New York, NY, USA, 13 pages. https://doi.org/10.1145/3448016.3452811

\section{INTRODUCTION}

Approximate set membership data sketches, such as Bloom and cuckoofi lters, allow users to query if an item $x$ belongs to a given set $\mathcal{S}$, i.e. if $x \in \mathcal{S}$. If $x \in S$, the item is always correctly classified. However, an item $x \notin S$ has some small probability of being incorrectly classified as being in the set. In other words, thefilters return no false negatives and have a small probability of returning false positives. These sketches are useful as they provide an inexpensive way to check whether an expensive operation needs to be performed. For example, in LSM-trees [41], a disk access can be skipped if a sketch guarantees a queried key is not in afi le. They are also used in join processing $[8,10,36]$ and cold storage structures [1] in databases and have many applications in networking [10].

We consider the general problem of testing set membership given predicates, in particular, equality predicates with an extension to range predicates. Consider a dataset $\mathcal{D}$ with each row consisting of a search key $k$ and vector of attributes a where the key may not

Permission to make digital or hard copies of part or all of this work for personal or classroom use is granted without fee provided that copies are not made or distributed for profit or commercial advantage and that copies bear this notice and the full citation on thefi rst page. Copyrights for third-party components of this work must be honored For all other uses, contact the owner/author(s).

SIGMOD '21, June 20-25, 2021, Virtual Event, China

(c) 2021 Copyright held by the owner/author(s).

ACM ISBN 978-1-4503-8343-1/21/06.

https://doi.org/10.1145/3448016.3452811 be unique. Given a predicate $\mathcal{P}$, we wish to test if an item $x$ is in the set $\mathcal{S}_{\mathcal{P}}$ of keys with attributes satisfying that predicate

$$
\mathcal{S}_{\mathcal{P}}=\{k:(k, a) \in \mathcal{D} \text { and } \mathcal{P}(a)=\text { true }\} .
$$

We show that this can have significant benefits in join processing by enabling predicates from one table to be pushed down to scans on other tables, thus reducing the number of tuples in intermediate results. Furthermore, unlike existing methods using approximate set membershipfi lters, this can be done using pre-builtfi lters that do not require additional table scans to constructfi lters at query time or communicating thefi lters over a network. We evaluate this reduction on the real world IMDB data set and show that the number of tuples that pass thefi lter is $<1 / 3$ the original number. Other possible applications of the sketch include problems in information retrieval and access control discussed in Section 9.

We propose the Conditional Cuckoo Filter (CCF) to address this problem. The CCF can be seen as a simple modification of a cuckoo hash table [49] where, rather than storing a key, value pair, it stores fingerprints or sketches of both. It is thus similar to cuckoofilters [20] which store only keyfi ngerprints. In a CCF, the value is a sketch of attribute columns. Using an attribute sketch greatly improves the functionality of thefi lter at a modest cost in space. A CCF differs from both cuckoo hashes andfi lters in that keys may not be unique in the CCF and require techniques to handle duplicates. This is since a CCF must store information about key, attribute pairs and not just a key. While existing cuckoo hash tables andfi lters only support inserting a small number of duplicated keys, we introduce a chaining technique that allows storage of additional duplicates.

The CCF supports two useful operations. Given an item $x$ and predicate $\mathcal{P}$, it tests if $x \in \mathcal{S}_{\mathcal{P}}$, in other words, if there is a matching row in the input data. Given just a predicate $\mathcal{P}$, some variations of the CCF return a cuckoofi lter for the set $\mathcal{S}_{\mathcal{P}}$. Like other approximate set membership sketches, it returns no false negatives.

\section{RELATED WORK}

A number of data sketches address the approximate set membership (ASM) problem. These include Bloomfi lters [6], d-left counting Bloomfi lters [7], quotientfi lters [4], and cuckoofi lters [20]. Several variants $[9,21,24,38,44,52,60]$, improve them through using cache awareness or compression, or add functionality to support counting. These sketches significantly reduce the amount of space required in practical regimes. Theoretical work [48] proposes a sketch that reduces the asymptotic space usage to the information theoretic minimum. In all these cases, the structures only address simple set membership queries with no notion of predicates.

These structures are used in databases to speed up a number of operations. Most related to our work are the joinfi lters used in Oracle [15, 37], Microsoft SQL Server [22], Informix XPS [61], and SAP ASE [54]. Given a set of dimension tables and a large fact table, 
joinfi lters construct Bloomfi lters when scanning the dimension tables and applying any predicates on them. Thesefi lters effectively push down predicates on dimension tables to the fact table scan and significantly reduce its output. Our work allows suchfi lters to be precomputed and stored. This allows thefi lters to be applied to dimension tables on the build side of the join. This can result in, for example, smaller hash tables which do not spill data to disk.

In databases, ASM sketches have been particularly useful for distributed join processing $[8,39,42,45]$ by reducing the number of tuples that must be loaded or sent across a network. They have also been used in join size estimation [46] and computing approximate join results [53]. ASM sketches are extensively used in log structured merge (LSM) tree based key-value stores [2, 3, 19, 23, 55] and in testing if query results are in a cache [47]. Outside of databases, approximate set membership sketches have a wide range of applications, particularly in networking [11, 58].

Also related are methods that sketch attribute columns. Column sketches [27] create small and hardware optimized data sketches that speed up scans involving a predicate. Bloom indexes in Postgres [25] similarly use Bloomfi lters on rows. A scan on the small sketch locates a subset of the full data that must be read.

For predicate pushdown methods, several methods search for hard constraints between columns in order to translate predicates on one column to a predicate on the join key. Such hard constraints include functional dependencies $[16,26]$, equivalent columns [12, $28,31]$, and magic sets $[17,56,57]$ and can be costly to search for. Recent work [30] infers cheaply computed zone maps [62] to map predicates on one column to coarsened range predicates on the join key at the partition level. However, each of these methods require strong interdependence of columns that is also of a particular form, and this dependence often does not exist in the data [56].

\section{FILTERS IN JOIN PROCESSING}

ASM sketches, such as Bloomfi lters, aid in reducing the cost of processing joins and semi-joins. Byfi ltering out tuples early in query execution, they can reduce the size of intermediate results and their associated downstream processing costs.

In a query plan with joins, each pairwise join is executed byfirst building a temporary structure such as a hash table or sorted array that 1) contains all tuples from one side or table of the join that potentially affect thefi nal output and 2) can be efficiently accessed by the join key. Then tuples on the other side can be efficiently matched and joined to entries in the temporary structure.

ASM sketches canfi lter out additional tuples at the veryfi rst step when scanning a table to build the temporary structure, thus potentially reducing the number of tuples in all downstream operations as well. This has several benefits. In memory limited scenarios, this can convert a Grace hash join that spills tuples to disk to a simple hash join that processes all tuples in memory [35]. In multi-way joins, reducing the size of intermediate pairwise join results also reduces downstream processing costs. In distributed settings, this can reduce the number of tuples sent across a network. In columnar stores, this can also avoid row stitching costs for output tuples [37].

To see how ASMfi lters reduce the number of tuples created, consider the following example that returns the production companies that have used dedicated production designers in a TV episode using the IMDB dataset.

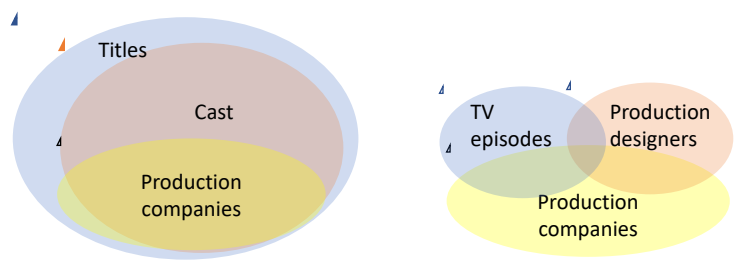

Figure 1: Left: Movie ids in the intersection of three tables are almost the same as ids in the production companies table. Right: If predicates restrict titles to TV episodes and cast to production designers then the intersection is small.

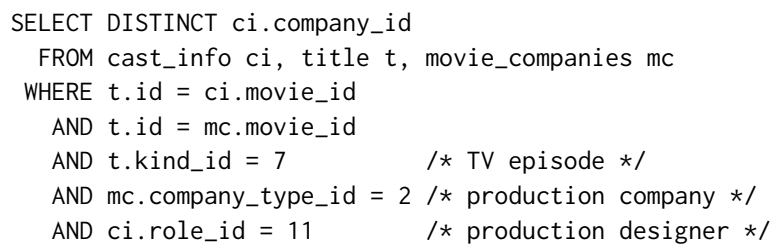

This query is a star join among three large tables with predicates applied to all three tables. Figure 2 shows a typical join tree for the query, executing pairwise joins $(m c \bowtie c i) \bowtie t$, and shows the number of tuples created in the 1) initial scan of $m c, 2$ ) the results of thefi rst pairwise join, and 3) thefi nal output of the entire join.

A typical execution engine can only apply predicates specified on $m c$ in the initial scan on $m c$ to build a hash table. Information in tables $t$ and $c i$ is not used. Likewise, thefi rst pairwise join $m c \bowtie c i$ cannot use of information in $t$. ASM sketches allow all three tables to be utilized at thefi rst step of scanning $m c$ and reduces the number of created tuples. The reduction naturally propagates to downstream operations. An ASM sketch on movie_id in ci, and likewise for $t$, can approximately restrict the hash table from the initial scan to tuples with movie_id in the intersection of all three tables. This can be beneficial when the intersection is much smaller than the table being scanned.

However, on the IMDB dataset described in Section 8 this intersection is large as all tables contain almost all movie_id's when no predicates are applied. Figure 1 shows a traditional ASM sketch provides little benefit since the intersection contains nearly the same movie ids as $m c$ alone, but if predicates can be applied to all tables, the size of the intersection can be greatly reduced. An ASM sketch that supports predicatefi lters can restrict tuples in the initial table scan on $m c$ to be approximately those in this small intersection. Figure 2 shows this ensures downstream operations also generate fewer tuples. Replicating this functionality with regular ASM sketches requires additional table scans that apply predicates, build ASM sketches, and, in distributed systems, transfer the sketches.

\section{PRELIMINARIES}

Our methods modify cuckoofi lters and hashes by adding sketched information about attribute values to each key. Since a single key can have multiple associated attribute vectors, multiset representations of the key are of particular importance. Before describing our methods, wefi rst review cuckoo hashing techniques. 


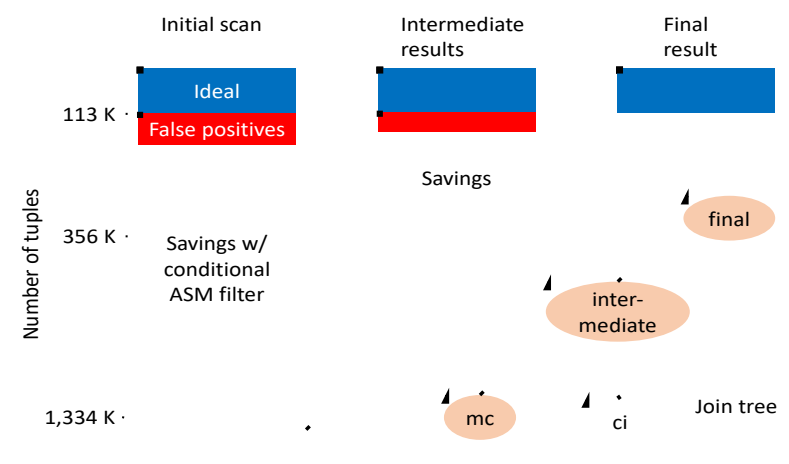

Figure 2: Top Left: Applying a conditional ASMfi lter reduces the number of tuples stored and created with savings that propagate downstream. Bottom right: Corresponding join tree with the stages of execution highlighted.

\begin{tabular}{c|l} 
Symbol & Meaning \\
\hline$h$ & Hash function \\
$(k, \mathbf{a})$ & Query for key $k$ and attributes a \\
$\ell, \ell^{\prime}$ & Bucket and alternate bucket for $k$ \\
$\kappa$ & Keyfingerprint \\
$\alpha$ & Attributefi ngerprint vector \\
$\beta$ & Load factor of hash table \\
$\oplus$ & XOR operation \\
$b$ & Number of entries per bucket \\
$d$ & max \# of duplicate keys in bucket pair \\
$H$ & Cuckoo hash table \\
$L_{\max }$ & Maximum chain length \\
$H_{\ell}, H_{\ell, i}$ & Set of entries, or entry $i$, in bucket $\ell$ \\
$m$ & Number of buckets in table \\
$(k, \mathbf{a}) \in H, k \in H$ & CCF returns true for query
\end{tabular}

\subsection{Cuckoo hashing}

Cuckoo hash tables [49] are a form of open addressing hash table. Such a hash table is arranged as afi xed size array of entries to avoid the overhead of storing pointers. Since hashed locations cannot grow in size, collision resolution techniques mustfi nd space among the remaining empty entries. This becomes increasingly difficult as the table reduces the space overhead of empty entries and the proportion offi lled entries, or load factor $\beta$, goes to 1 . For example, linear probing has an expected query and insertion cost of $O(1+$ $\left.1 /(1-\beta)^{2}\right)$ that grows quickly as $\beta$ approaches 1 .

Unlike collision resolution techniques where the locations of items in the hash table are immutable after insertion, cuckoo hashing can relocate items when there are collisions at insertion time. By resolving collisions at insertion time, the number of buckets probed at query time can be reduced to two while still being able to achieve a high load factor on the table. Furthermore, cuckoo hash tables have $O(1)$ amortized expected insertion time. Cuckoo hash tables are typically arranged in a tiered fashion so that an item is first hashed to one of $m$ candidate buckets. Each bucket contains $b$ entries in which data can be stored.

The fundamental operations in cuckoo hashing are the insertion and kick out operations. When inserting an item, value pair $(k, v)$, cuckoo hashing hashes the key to a choice of two possible buckets $\ell, \ell^{\prime}$ rather than being constrained to one choice. If $k$ is already in one of the buckets, the value is simply updated. Otherwise, if either bucket is not full, the pair is added to a non-full bucket. If both are full, it picks a random pair $\left(k^{\prime}, v^{\prime}\right)$ from the two buckets. It "kicks out" that pair and replaces it with $(k, v)$. Since there are two bucket choices, the pair $\left(k^{\prime}, v^{\prime}\right)$ is then reinserted into the sketch at its alternate bucket. The process of kicking out and reinserting is performed up to some maximum MaxKicks number of times. If the process reaches this maximum then the table is resized and all pairs are reinserted. To query a cuckoo hash table, only the two possible buckets need to be examined for the key.

\subsection{Cuckoofilters}

Cuckoofi lters adapt cuckoo hash tables for key-only approximate set membership queries rather than key-value queries. There are two primary differences from cuckoo hash tables. First, to save space, a cuckoofi lter stores only a smallfingerprint $\kappa$. This is a hash of the key $k$ into a small number of bits. It does not store the full key nor any value associated with the key. Second, it uses partialkey cuckoo hashing where the alternate bucket $\ell^{\prime}=\ell \oplus h(\kappa)$ is determined only by the bucket $\ell$ and thefi ngerprint. Unlike cuckoo hashing which requires the full key, the alternate bucket can be computed using just the keyfi ngerprint stored in the sketch.

To check if a key $k$ exists, the items in its 2 buckets $\ell, \ell^{\prime}$ are checked for a matchingfi ngerprint. Trivially, if $k$ was inserted before then thefi lter willfi nd a match, so there are no false negatives. If $k$ was not previously inserted, there is some probability of a false match due to random hash collisions on thefingerprint.

For a typical setting where the number of entries per bucket is $b=4$, an optimally sized cuckoofi lter requires approximately $\left(\log _{2} 1 / \rho+3\right) / \beta$ bits per item to achieve a desired FPR of $\rho$ where $\beta$ is the load factor of the table. According to [20], an optimally sizedfi lter empirically achieves $\beta \approx 95 \%$ when $b=4$. Compared to Bloomfi lters which require $\approx 1.44 \log _{2} 1 / \rho$ bits per item to achieve the same FPR, an optimally sized cuckoofi lter requires fewer bits per item when the desired FPR $\rho<0.35 \%$. Additional semi-sorting optimizations can further reduce the space so that they are smaller than an optimally sized Bloomfi lter when the target FPR $\rho<2.5 \%$.

\subsection{Multisets}

A conditional ASM sketch that supports predicates effectively stores a multiset rather than a set. It contains information for a set of key-attribute pairs $(k, a)$. Since the key is not necessarily unique, the collection of search keys $k$ forms a multiset. Existing cuckoo filters have limited support for multisets by allowing duplicate copies of a keyfi ngerprint to be inserted. While this makes cuckoo filters morefl exible than Bloomfi lters as they support deletions by removing a copy of a keyfi ngerprint, at most $2 b$ copies of a key can be inserted into its two buckets. Furthermore, there are no theoretical guarantees that a high load factor can be obtained. Empirically, we see they cannot. Figure 3 shows the load factor for a plain cuckoofi lter decreases dramatically when there are duplicate keys, and insertions into thefi lter can fail almost immediately when the distribution of duplicated keys is highly skewed. Cuckoofilters can also be extended by adding a counter to each entry, but this approach cannot store attribute information. 


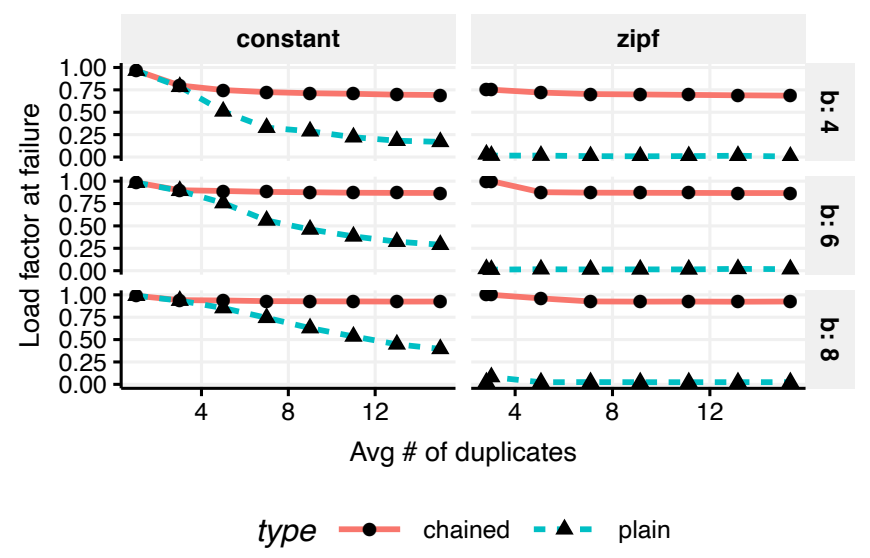

Figure 3: Chaining enables high load factors. A regular cuckoofi lter achieves reasonable load factors only when the maximum number of replicates for a key is small.

\section{CONDITIONAL CUCKOO FILTERS}

We now introduce the Conditional Cuckoo Filter (CCF). These support set membership queries with equality predicates. Like a cuckoofi lter, a CCF is based on cuckoo hashing and saves space by usingfi ngerprints or sketches. By storing sketches for both key and attribute values rather than just the key, they provide a space efficient structure that supports predicates. This can be much more efficient than the alternative: storing a separate ASM sketch for each combination of predicate values, causing exponential growth in size with the number of predicates.

There are two main problems in adding back this attribute information. First, how can a set of attributes be summarized in a small amount of space? Second, how can the cuckoo hash table handle non-unique keys, especially when the key distribution is skewed?

We propose and evaluate several solutions to these problems. To solve the former, wefi rst introduce the extremely simple, but novel, idea of sketching attributes and provide three ways of sketching attributes: using a vector offi ngerprints, a Bloomfi lter, or a mixture of the two. Next, we develop two novel extensions to cuckoofilters and hashing in order to allow for more duplicate keys. We introduce a chaining mechanism which allows the CCF to use more buckets as more duplicates are encountered and a method to switch from fingerprint vectors to Bloomfilters.

\subsection{Attributefi ngerprint vectors}

The simplest method to sketch an attribute vector hashes each attribute value into a small number of bits $s$, say 4 or 8 , to construct a vector of attributefi ngerprints that is significantly smaller than the original data. Despite their small size, these attribute sketches can be effective in applications. For example, in join processing, the FPR does not need to be extremely low to be effective. The output size after applying an equality predicate to an intermediate scan is

$$
\begin{aligned}
\mathbb{E} M_{\text {output }} & =M_{\text {true }}+\mathbb{E} F P R \cdot\left(M_{\text {original }}-M_{\text {true }}\right) \\
& <M_{\text {true }}+\mathbb{E} F P R \cdot M_{\text {original }}
\end{aligned}
$$

where $M_{\text {original }}$ is the total number of tuples in the original table, $M_{\text {true }}$ is the number of true matching tuples, and $M_{\text {output }}$ is the number of tuples that match according to the CCF. Thus, when the number of true matching tuples is a small percentage of the output tuples, $M_{\text {true }} / M_{\text {out put }} \approx 0$, a relatively poor FPR of just $10 \%$ reduces the number of tuples produced by a factor of nearly 10 . Furthermore, if more than one predicate is applied, the reduction can be multiplicatively amplified. Similarly, there is little reason to target a FPR much smaller than $M_{\text {true }} / M_{\text {original }}$, as this unavoidable cost becomes the dominant cost in processing.

The resulting CCF will thus have a very low FPR if a key is absent from the set and a higher FPR if the key exists but there is no matching attribute. We use a, $\alpha$ to denote an attribute vector and itsfi ngerprint vector. When using attributefi ngerprint vectors, the underlying cuckoo hash table must be modified to handle duplicated keys with unique attributefingerprints.

\subsection{Bloomfi lter attribute sketches}

A second choice represents attributes with a Bloomfi lter. Each (attribute name, value) pair is inserted into a small Bloomfi lter. The resulting sketch is simply a cuckoofi lter with an added Bloomfilter for each entry. Algorithm 1 summarizes the procedure for querying thefi lter. The only difference from a regular cuckoofi lter query is the additional check to verify that the query predicate matches the attribute sketch, namely all attributes in the query predicate are found in the Bloomfi lter A CCF using Bloom attribute sketches can also support queries that only specify a predicate and not a key. It returns a cuckoofi lter which a downstream process can use to check the existence of a key. To do this, simply erase all entries where the predicate does not match and return the resulting array of keyfi ngerprints. This is summarized in Algorithm 2.
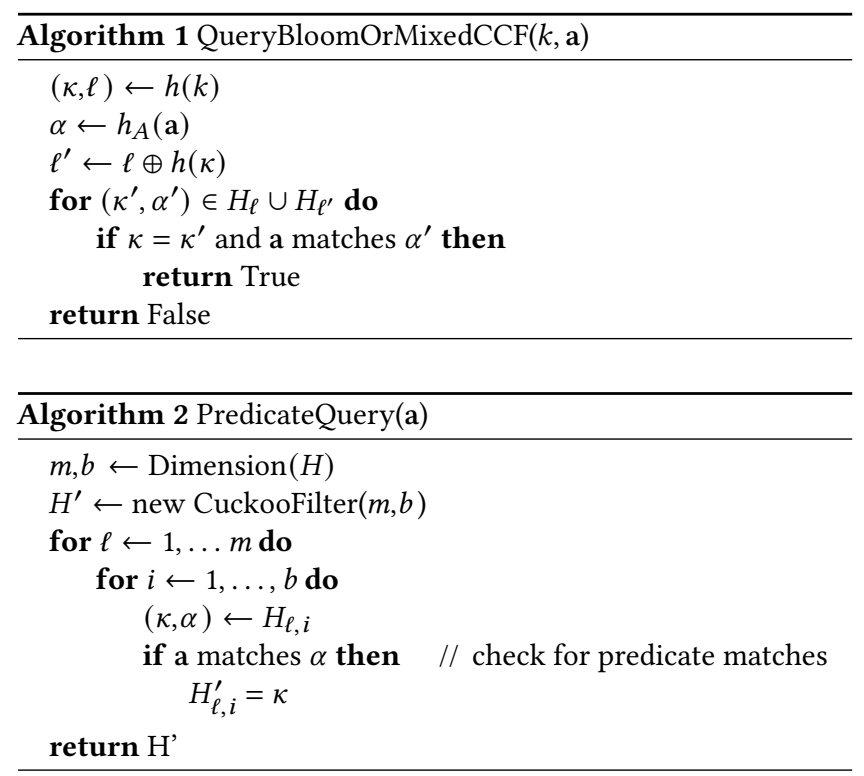

Using a Bloomfi lter attribute sketch has mixed effects on the size of the sketch and the FPR. First, note that the occupied entries in the sketch are exactly the same as those of a cuckoofi lter. Thus, appropriately sized filters are theoretically guaranteed [18] to obtain high load factors with high probabilty. For our other methods, we only have empirical results showing high load factors are obtained. However, a Bloomfi lter is less bit efficient than afingerprint 
vector. An optimized Bloomfi lter requires $\approx 1.44 \log _{2}(1 / \rho)$ bits per attribute to achieve an FPR of $\rho$ versus $\log _{2}(1 / \rho)$ for a fingerprint vector. This inefficiency is exacerbated since it is not possible to choose optimal parameters for the Bloomfi lter. The optimal choice for the number of hash functions to use depends on the number of distinct (attribute, value) pairs that will be added to thefi lter. These are not known in advance and can vary greatly across keys. Second, when multiple rows of data share the same key but have different attribute vectors, a Bloomfi lter attribute sketch does not encode which attribute values occur together in the same row. If two rows contain the query key with attribute vectors $\left(a_{1}, a_{2}\right),\left(a_{1}^{\prime}, a_{2}^{\prime}\right)$, the predicate $A_{1}=a_{1} \wedge A_{2}=a_{2}^{\prime}$ has no matching row, but a Bloom filter attribute sketch is guaranteed to return a false positive. This remains true if the Bloomfi lter was replaced with any other ASM sketch. On the other hand, if all queries contain only a single equality predicate, thenfi ngerprint vectors can unnecessarily store a single attribute value $A_{1}=a_{1}$ multiple times when the second attribute $A_{2}$ is varying. A Bloomfi lter only encodes it once.

\subsection{CCF Multiset representations}

One advantage of attributefi ngerprint vectors is the ability to store co-occurence information. This comes at the cost of a single key occupying multiple entries in the CCF if if it has multiple attribute vectors that differ in any way. The ability to handle a relatively large number of duplicates is an important ability that normal cuckoo hash tables lack. A key's two buckets contain at most $2 b$ entries, and inserting any more copies of a key is guaranteed to fail. This is problematic as many data sets have highly skewed key distributions. Existing ASM sketches that support duplicates [20, 50] either fail after the buckets are full or only maintain a counter that cannot store attribute information.

We present two strategies to address this and ensure no false negatives are produced. One converts attributefi ngerprint vectors to Bloomfi lters when too many duplicates are encountered. We refer to this approach as Mixed. The second is a form of chaining that allows a key to utilize more than 2 buckets. In both cases, we allow a maximum of $d$ duplicated keyfi ngerprints per bucket pair. If an insertion for key $k$ is on a bucket pair already containing $d$ copies of thefingerprint $\kappa$, then either the $d$ copies are converted to a Bloomfi lter attribute sketch or additional bucket pairs are considered via the chaining procedure. We are not aware of prior work on chaining multiple buckets in cuckoofi lters, though [60] chains entire cuckoofi lter tables. Chaining tables increases the total capacity of afi lter but forces the sketch to be large if there is skew in the key distribution. We note that although previous works $[7,21,50]$ address multiset representations, they do so via counters that cannot store predicate information.

\subsection{Bloomfi lter conversion}

Consider a key $k$ and attribute vector a to be inserted in the bucket pair $\ell, \ell^{\prime}$. Let $|\kappa|,|\alpha|$ denote the size of the keyfi ngerprint and attributefi ngerprint vectors and $\# \alpha$ denote the number of attributes. Suppose there are already $d$ copies of thefingerprint $\kappa$ in the $2 b$ entries of $\ell, \ell^{\prime}$. Bloomfi lter conversion takes the $d|\alpha|$ bits currently used to store $d$ fingerprint vectors and constructs a single Bloom filter in their stead. Each sketch entry also requires an additional bit to track if it contains a Bloomfi lter or afi ngerprint vector.
This conversion operation has the advantage that it can never fail. However, it adds complexity in storing a Bloomfi lter among $d$ entries and maintaining it whenever a bucket's entry is kicked into the alternate bucket. It has the same advantages and disadvantages outlined in Section 5.2 when directly using a Bloomfi lter attribute sketch but with two main differences. It has a further disadvantage in that hash collisions can be introduced both from hashing attribute values intofi ngerprints and from insertingfingerprints into the Bloomfi lter. Directly using a Bloomfi lter only introduces collisions from the latter. It has an advantage in that the Bloom filter parameters can be chosen more easily since the minimum number of duplicates is $d$. The Bloomfi lter parameters do not need to be optimized to also handle rows with a unique key.

The storage of the entries can be further optimized to avoid storing the same keyfi ngerprint multiple times. Instead, each bucket can store a single copy of the keyfi ngerprint along with the number of entries the Bloomfi lter attribute sketch occupies in that bucket. If the other entries sharing the same keyfi ngerprint are stored contiguously, then the Bloomfi lter can be successfully reconstructed. In this case, the required number of bits to store thefingerprint and counts is $2\left(|\kappa|+\left\lceil\log _{2} d\right\rceil\right)$ and the number of bits in $d$ entries is $d(|\kappa|+|\alpha|+1)$.

We choose the number of hash functions to be approximately the optimal number assuming there are $(d+1) \cdot \# \alpha$ unique attribute (name, value) pairs added to thefi lter. Let $|B|$ be the number of bits available to the Bloomfi lter.

$$
\begin{aligned}
\# \text { hashes } & \approx \frac{|B|}{(d+1) \cdot \# \alpha} \log 2 \\
& \approx \frac{|\alpha|}{\# \alpha} \frac{d}{d+1} \log 2 \quad \text { if }|\alpha| \gg|\kappa|
\end{aligned}
$$

Algorithm 3 summarizes the method to convert attributefingerprint vectors to a Bloomfi lter. Figure 4 shows howfi ngerprints and attributes are stored for one bucket in the CCF.

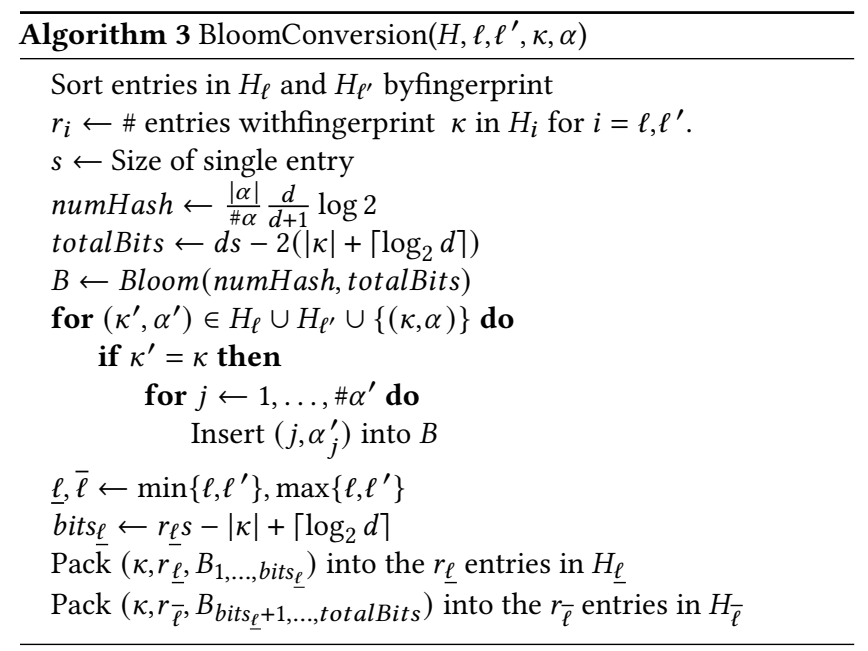

\subsection{Chaining}

Chaining introduces additional bucket pairs whenever an insertion would violate the constraint that at most $d$ copies of a keyfingerprint $\kappa$ are in a bucket pair $\ell, \ell^{\prime}$. A second bucket pair is determined 
bucket

\begin{tabular}{|l|l|l|l|}
\hline $\begin{array}{l}\text { finger- } \\
\text { print }\end{array}$ & Attribute sketch & $\begin{array}{l}\text { finger- } \\
\text { print }\end{array}$ & Attribute sketch \\
\hline
\end{tabular}

Figure 4: Fields in one CCF bucket with 2 entries per bucket. In Bloomfi lter conversion, the grey area may be repurposed as a single Bloomfi lter, and one additional bit indicates if the conversion is performed.
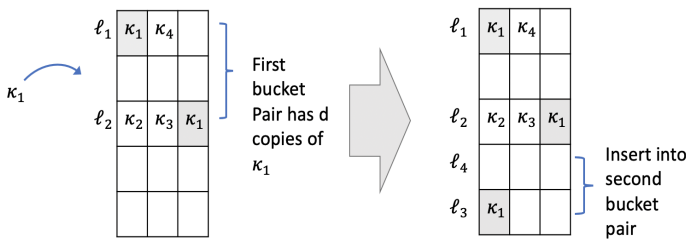

second
bucket

pair

Figure 5: Illustration of chaining procedure

by hashing the bucket pair andfingerprint, $\tilde{\ell}:=h\left(\min \left\{\ell, \ell^{\prime}\right\}, \kappa\right)$ and $\tilde{\ell^{\prime}}:=\tilde{\ell} \oplus h(\kappa)$. This can be inductively applied to generate a sequence of bucket pairs, which we cap at $L_{\max }$ pairs. By using only information about the original key $k$ stored in the $\mathrm{CCF}$, namely the unordered bucket pair $\left\{\ell, \ell^{\prime}\right\}$ andfingerprint $\kappa$, this procedure generates the same sequence of bucket pairs during a cuckoo kickout, that has no access to $k$, as a cuckoo insertion or query with access to $k$. The min can also be replaced by any symmetric function.

A query or insertion searches for a match or until thefi rst nonfull bucket pair where a full bucket pair is one containing $d$ copies of thefingerprint $\kappa$. If all $L_{\max }$ bucket pairs are checked and full, then an insertion discards the insertion item, and a query returns true regardless of the predicate to avoid false positives. The query algorithm is summarized in Algorithm 5. An insertion is illustrated in Figure 5 and summarized in Algorithm 4. Correctness is proven by the following lemmas and theorem.

Lemma1. Consider a CCF with chaining. Given any keyfingerprint $\kappa$ and bucket $\ell$, let $\ell^{\prime}=\ell \oplus h(\kappa)$ be the alternative bucket. The total number $c_{\kappa}(t)$ of copies of $\kappa$ in buckets $\ell, \ell^{\prime}$ after $t$ insertions is increasing and capped by the parameter $d$.

Proof. We prove this by induction. This trivially holds in the base case where the CCF is empty. $c_{\kappa}(t)$ can only decrease if an entry containing $\kappa$ is kicked out in a cuckoo kick operation. Assume WLOG that a copy of $\kappa$ in bucket $\ell$ was kicked out at the $t+1$ insertion. Suppose $c_{\kappa}(t)<d$. After the kickout there are still $<d$ copies of $\kappa$ in $\ell, \ell^{\prime}$ and the kicked out copy must be reinserted in these buckets, so $d \geq c_{\kappa}(t)+1 \geq c_{\kappa}(t+1) \geq c_{\kappa}(t)$. Now suppose $c_{\kappa}(t)=d$. If the newfingerprint $\kappa^{\prime} \neq \kappa$, then after the kickout there are fewer than $d$ copies of $\kappa$ and it must be reinserted into the same bucket pair. Otherwise $\kappa^{\prime}=\kappa$, and the insertion must choose a new bucket pair. Since $\ell^{\prime}=\ell \oplus h(\kappa)$, any new bucket pair must either be identical to $\left(\ell, \ell^{\prime}\right)$ and another bucket pair must be generated, or it is disjoint. In both cases, the bucket pair $\ell, \ell^{\prime}$ is unaffected so $c_{\kappa}(t)=c_{\kappa}(t+1)=d$.

LemMA2. Let $(k$, a) be a key, attribute vector pair with correspondingfingerprints $(\kappa, \alpha)$ and $H$ be a CCF with chaining. There is afi xed sequence of buckets $\ell_{1}, \ell_{2}, \ldots \ell_{n}$ determined by $k$ with $n \leq 2 L_{\text {max }}$ that it can be inserted into. Furthermore, if at any time it is in $\ell_{i}$, then all pairs $\ell_{2 j}, \ell_{2 j-1}$ with $2 j-1<i$ containd copies of $\kappa$.
Proof. The sequence is recursively defined by $\ell_{1}=h_{\ell}(k), \ell_{2 j}=$ $\ell_{2 j-1} \oplus h(\kappa)$ and $\ell_{2 j+1}=h_{b}\left(\min \left\{\ell_{2 j-1}, \ell_{2 j}\right\}, \kappa\right)$. It ends if there is a cycle or the maximum chain length of $L_{\max }$ bucket pairs is reached. This sequence is deterministic since $\ell$ and $\kappa$ are deterministic functions of $k$. A $\kappa, \alpha$ pair is inserted in thefi rst bucket pair in the chain with fewer than $d$ copies of $\kappa$. A reinsertion after a kickout operation from bucket pair $\ell_{2 i}, \ell_{2 i+1}$ does the same starting from $\ell_{2 i}$. Thus, each pair $\ell_{2 j}, \ell_{2 j-1}$ with $2 j-1<i$ contain $\geq d$ copies of $\kappa$. Lemma 1 shows there are exactly $d$ copies.

THEOREM3. A CCF with chaining and no failed insertions returns no false negatives.

Proof. Given a key $k$ and predicate $\mathcal{P}$, Let $k$, a be some matching row withfingerprints $\kappa, \alpha$. If no rows with key $k$ are discarded, then by Lemma 2 , all bucket pairs before the actual location corresponding to $k$, a contain exactly $d$ copies of $k$ 'sfingerprint $\kappa$. Otherwise, by Lemma 1 , all $2 L_{\max }$ buckets in the bucket chain for $k$ contain $d$ copies of $\kappa$. Since a query for $k, \mathcal{P}$ will continue searching bucket pairs along the chain for $k$ when there are $d$ copies of $k$ 'sfingerprint $\kappa$ in the bucket pair, it will eitherfi nd a matching entry or reach the maximum chain length. Both conditions return true.

Note that generating alternative bucket pairs may create a cycle of pairs. In this case, the insertion procedure will not fail but will not generate $L_{\max }$ unique bucket pairs. Such cycles can be detected using Floyd's cycle detection algorithm and the chain can be extended using the same chaining procedure with a different hash function for each cycle detected but taking the minimum bucket in the entire chain rather than bucket pair.

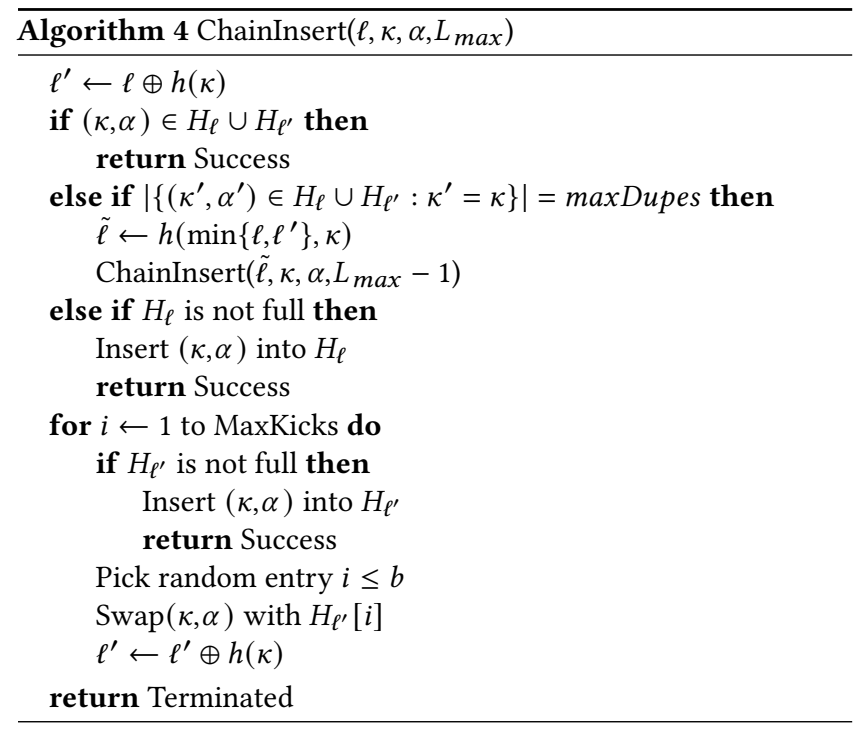

Like Bloomfi lter attribute sketches, the chaining method can support predicate only queries. However, it cannot simply erase entries with non-matching attribute values. This could introduce gaps in a chain where some bucket pair does not contain $d$ copies of afi ngerprint. A query could stop probing bucket pairs early and yield false negatives. Instead, the sketch must keep the key fingerprint and add a bit to mark the entry as non-matching. 


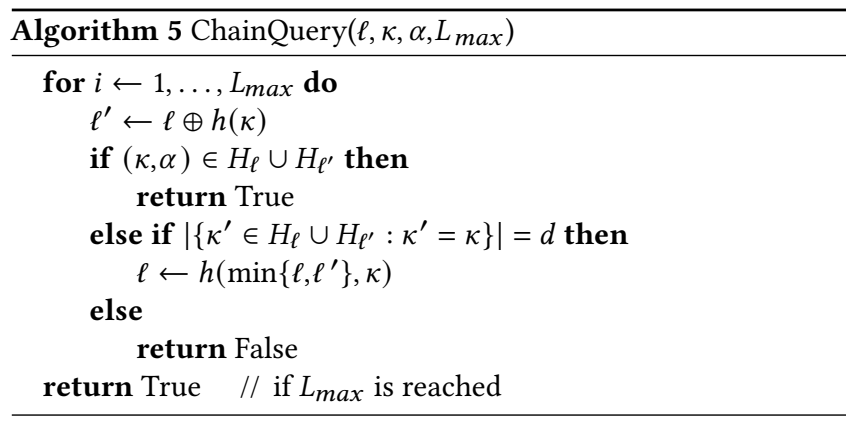

\begin{tabular}{l|ccc|l} 
& \multicolumn{3}{|c|}{ Queries } & \# non-empty entries \\
Filter & $k$ & $(k, \mathcal{P})$ & $\mathcal{P}$ & (upper bound) \\
\hline Cuckoofilter & $\checkmark$ & & & $n_{k}$ \\
CCF w/ Bloom & $\checkmark$ & $\checkmark$ & $\checkmark$ & $n_{k}$ \\
CCF w/ conversion & $\checkmark$ & $\checkmark$ & $\checkmark$ & $n_{k} \mathbb{E} \max \{A, d\}$ \\
CCF w/ chaining & $\checkmark$ & $\checkmark$ & & $n_{k} \mathbb{E} \max \left\{A, d L_{\text {max }}\right\}$
\end{tabular}

Table 1: Supported queries and sizing for different CCFs. $n_{k}$ is the number of distinct keys and $A$ is the number of distinct attribute vectors associated with a randomly chosen $k$.

\section{ANALYSIS}

The accuracy of CCF's and other approximate set membership sketches can be measured by the false positive rate (FPR). Unlike regular cuckoofi lters, the FPR for CCF's is not a constant. Queries can result in false positives due to spurious matches on the key fingerprint, on the attribute sketch, or both. Because of this, the FPR depends on the distribution of the underlying data and the query itself. We provide some simple bounds on the FPR expressed in these relevant quantities.

\subsection{False positive rate: Key only queries}

For a standard set membership query for a key with no predicates, the CCF has a FPR similar to a regular cuckoofi lter. When using Bloom filter attribute sketches, the CCF is, in fact, identical to a cuckoofi lter when the attribute sketches are ignored. Thus, they have exactly the same FPR as cuckoofi lters on key only queries. We show that all the variations of the CCF are governed by a bound of the same form for key only queries.

The typical bound on the FPR on a cuckoofi lter is given by a union bound. A key $k$ that was not inserted into the sketch generates a random keyfingerprint $\kappa$ with $|\kappa|$ bits. The probability that $\kappa$ randomly matches any given entry in the sketch is $2^{-|\kappa|}$. Summing over the entries that can be probed by a query gives a union bound on the FPR. A slight refinement of the typical bound for the FPR sums this probability for a spurious match over non-empty entries rather than all $2 b$ entries in the key's bucket pair. Letting $D$ be this number of non-empty entries, this gives a bound on the FPR for key only queries using Bloom attribute sketches as

$$
F P R^{k e y} \leq \mathbb{E} D 2^{-|\kappa|}
$$

For a CCF using Bloom conversion, the bound holds with $D$ representing the number of distinct keyfi ngerprints in a bucket pair.

For a CCF using chaining, lemma 2 shows that the chain is irrelevant for key only queries. Only thefi rst bucket pair must be checked. Thus, the FPR formula is also the same as above. This result is of interest since although insertions can probe up to $2 L_{\max }$ buckets, there is no penalty for probing more buckets at query time.

\subsection{FPR: Key and predicate queries}

CCF's are distinguished by their ability to answer queries for a key and predicates. Consider a query for a key $k$ and equality predicates $A_{i}=a_{i}$ for some set of attributes $i \in \mathcal{I}$. Further suppose there are no matches on the full data so that a positive return value is a false positive. The probability that a CCF returns true can be decomposed as the probability of matching the key $p(k \in H)$ times the probability of matching the predicate given the key is found $p(\mathscr{P} \in H[k] \mid k \in H):$

$$
p(\text { false positive for }(k, \mathcal{P}))=p(k \in H) p(\mathcal{P} \in H[k] \mid k \in H) .
$$

Here, $H$ denotes the keyfi ngerprints in thefi lter, and $H[k]$ denotes the entries probed by a query involving $k$ and which contain the keyfingerprint $\kappa . \mathcal{P} \in H[k]$ denotes that there is a match for the predicate among those entries. Consider the following cases

- The key $k$ is not in the data

- The key $k$ is in the data, but there is no matching predicate In the former case, the FPR is trivially bounded by $p(k \in H)$ which is upper bounded in the previous subsection. The FPR is lower if there are any predicates that are false for a key. An upper bound on the FPR of $\leq 5 \%$ can be achieved with a keyfi ngerprint size of 8 and 6 buckets per entry, holding for all variations of the CCF.

In the latter case, a false positive only occurs when there is a spurious match for the predicate $\mathcal{P}$ among the entries in $H[k]$ since $p(k \in H)=1$. The FPR depends on the attribute sketch used. For Bloomfi lter attribute sketches, the FPR depends on the number of distinct attribute values $z_{k}$ inserted into the small Bloomfi lter for key $k$ as well as the number of predicates $u$ for which the Bloom filter should return false. The FPR is given by

$$
p_{\text {Bloom }}(\mathcal{P} \in H[k])=\rho\left(z_{k}\right)^{u}
$$

where $\rho\left(z_{k}\right)$ is the FPR of a Bloomfi lter with $z_{k}$ entries added into it. The FPR thus decreases exponentially quickly as there are more unmatched attributes. Also note that when query predicates on different attributes match on different rows, $u$ can equal 0 , and the FPR can be 1 as described in Section 5.2.

When using attributefi ngerprints, the FPR depends on the number of duplicate keys inserted, the attributefi ngerprint length $|\alpha|$, and the number of predicates that are false on each row. The probability the $i^{t h}$ entry in the CCF with keyfingerprint $\kappa$, denoted by $H[k]_{i}$, triggers a false positive is $p\left(\mathcal{P} \in H[k]_{i}\right)=\tilde{\rho}^{\tilde{u}_{i}}$ where $\tilde{u}_{i}$ is the number of non-matching predicates on the input data row corresponding to entry $H[k]_{i}$ and $\tilde{\rho}=2^{-|\alpha|}$ is the probability of a collision in thefi ngerprint. Summing over the false positive probabilities for the entries gives an upper bound on the FPR

$$
p_{\text {chained }}(\mathcal{P} \in H[k]) \leq d L_{\text {max }} \mathbb{E} 2^{-|\alpha| \tilde{U}}
$$

since there are at most $d L_{\max }$ entries for key $k$. Here, $\tilde{U}$ denotes a randomly chosen $\tilde{u}_{i}$.

For Bloom conversion, the FPR depends on whether the entry for the key $k$ has been converted to a Bloomfi lter. Although the formulae are upper bounds on expected FPR, Figure 6 shows they are reasonably good predictors of actual FPR. 


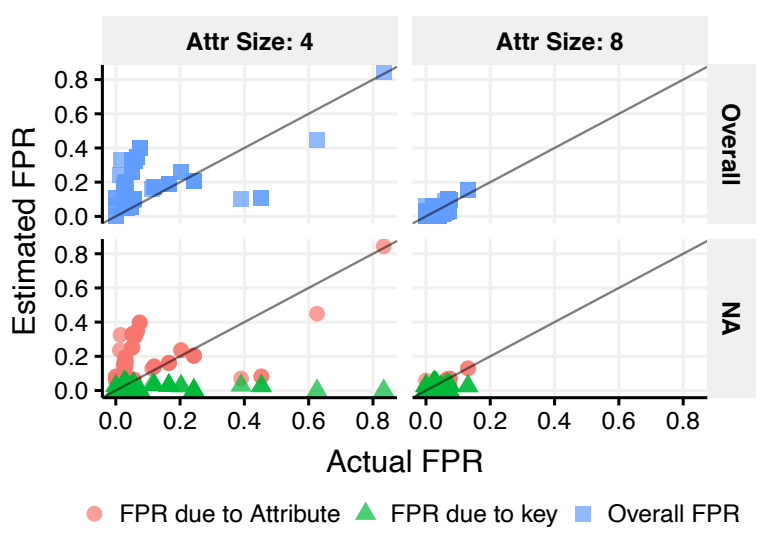

Figure 6: The bounds on the expected FPR are good predictors of the actual FPR when using attributefingerprints.

\subsection{Size and parameter choice}

Conditional cuckoofi lters have more parameters than regular cuckoo filters. Cuckoofi lters only require setting the number of buckets $m$ and the number of entries per bucket $b$. CCF's can additionally require setting the maximum number $d$ of duplicates per bucket pair, $L_{\max }$ the maximum chain length, and any additional parameters required by the attribute sketch.

We derive an upper bound on the number of non-empty entries and experimentally show the attainable load factor is not sensitive to the underlying data. Together these can be used to size the sketch. These bounds and constants depend on parameters $d$ and $L_{\max }$ which affect the number of duplicates stored in the sketch.

Denote the total number of distinct keys by $n_{k}$ and the number of non-zero entries in a CCF by $Z^{c}$. Since a CCF with Bloom attribute sketches has the same non-empty entries as a regular cuckoofi lter, the number of non-zero entries can be upper bounded by $n_{k}$. For other cases, let $r_{k}$ be the number of duplicates for key $k$ that have distinct attribute values. Bloomfi lter conversion will allocate a maximum of $\max \left\{d, r_{k}\right\}$ entries for that key. Let $A=r_{X}$ for a randomly chosen key $X$. Then the expected number of used entries is bounded by $\mathbb{E} Z^{c} \leq n_{k} \mathbb{E} \min \{A, d\}$. Similarly, a CCF with chaining uses at most $d L_{\max }$ entries for a single key, so the expected number of entries used is bounded by $\mathbb{E} Z^{c} \leq n_{k} \mathbb{E} \min \left\{A, d L_{\max }\right\}$. These sizes are summarized in Table 1. Figure 7 shows the bound on the number of entries closely matches the actual number needed.

From experiments on chaining, wefi nd a reasonable rule of thumb sets the number of entries per bucket to $b \approx 2 d$. This way, at least 4 distinct keys can be stored in a bucket pair $\ell, \ell^{\prime}$. Typically more keys can be stored since only 1 keyfi ngerprint will use that particular pair of bucket locations. This provides relatively high load factors while ensuring buckets are not costly to scan. Figure 3 shows that a setting of $b=4$ that is typical for cuckoofi lters achieves a load factor of around $\beta \approx 75 \%$ regardless of the number of duplicate keys. A slightly larger value of $b=6$ achieves a load factor close to $\beta \approx 87 \%$ even when there are many duplicates. An appropriate estimate for the required size for a CCF is $m \cdot b \approx \mathbb{E} Z^{c} / \beta$.

When the sketch is optimally sized, Figure 8 shows that lower settings for $d$ tend to achieve better use of bits. This is primarily

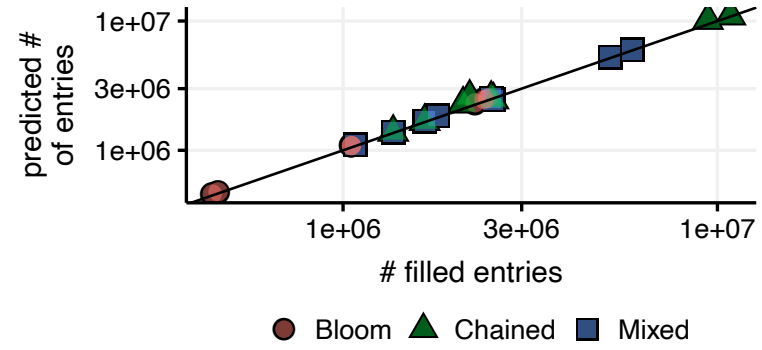

Figure 7: The predicted number of entries needed for each filter closely match the actual number for the workload.

due to smaller values of $d$ yielding higher load factors. Given afi xed setting for the number of bins $m$ and entries per bin $b$, we found the best $d$ is the largest under which all insertions pass. We chose $d=3$ which provides small bucket sizes and a good load factor.

\subsection{Attribute sketch parameters}

Figure 11 shows the performance of different CCF's under various parameter choices. Generally, we also found increasing the attribute sketch size more beneficial than increasing the keyfi ngerprint size. Figure 6 shows that at small attribute sketch sizes, the false positives are primarily due to bad matches on the attribute sketches. In this case, a false positive $k$, a is ascribed to a key if the key is not in the sketch, in other words, $k \notin H$. Otherwise, it is easily ascribed to the attribute. We also found small values for the number of hash functions used by Bloomfi lters to be preferable as it does not becomefi lled with ones too quickly.

\section{RANGE QUERIES}

Conditional Cuckoofi lters can be extended to support range predicates. Given a column with a range predicate, one simple method is to bin the column into a small number of bins. A range predicate can then be converted into a small in-list. The disadvantages of this approach are that the binning process introduces error and that long ranges must check more bins. Since each bin that does not contain a true match can return a false positive, both can increase the FPR. Another method uses a standard approach of using a dyadic expansion over the range $\left[a_{0}, b_{0}\right]$ of the column. An item $x$ can be represented as a sequence of intervals $\left[a_{1}, b_{1}\right], \ldots\left[a_{\eta}, b_{\eta}\right]$ with exponentially decreasing lengths $b_{i+1}-a_{i+1}=\left(b_{i}-a_{i}\right) / 2$ down to somefi nal granularity $b_{\eta}-a_{\eta}$. This requires $\eta$ insertions into a $\mathrm{CCF}$ for each item, and a range query likewise requires querying for the existence of up to $\eta$ intervals that cover the range. This is primarily useful when the number of desired intervals is large since $\eta$ has a logarithmic dependency on the number of intervals. We use the simpler binning in our experiments since they use few bins.

\section{EXPERIMENTS}

We consider two experiments. Thefi rst "multiset experiment" uses synthetic data to examine the ability of the chaining procedure to store duplicate keys while obtaining a high load factor. The second "JOB-light" experiment examines the ability of the CCF to reduce output sizes on a join benchmark on real data [33]. 


\subsection{Multiset experiment data and setup}

Data: Our experimental results show chaining dramatically improves the ability of a cuckoofi lter to handle duplicate keys. We simulated key frequencies using either a truncated Zipf-Mandelbrot distribution or a stream where every key has the same number of duplicates. We do not generate attribute vectors for this experiment. For the Zipf-Mandelbrot distribution with a mass function of the form $p(x) \propto(c+x)^{-\alpha}$, wefi $\mathrm{x}$ the offset $c$ to the Mandelbrot parameter $2.7^{1}$ and truncate the range to be in $[1,500]$. For both distributions, we generate multiple datasets, each with a different number of average duplicates per key.

Setup: For CCF parameters we vary the number of entries $b$ per bucket from 4 to 8 and follow the rule-of-thumb in Section 6.3 in setting the maximum number of duplicates per bucket pair to $d=3$. Figure 8 shows this choice also has close to optimal efficiency. The maximum number of bucket pairs for a key $L_{\max }=$ $\infty$ is uncapped. For each experimental run, we generate a dataset that is approximately $20 \%$ larger than the capacity of the sketch and measure the number of items processed before thefi rst failed insertion and the load factor at that point. A failed insertion is thefi rst time a new key, attribute pair fails to generate a new entry in the sketch. The order of items is randomly permuted. We use the additional cycle detection and resolution method in these experiments. The results are averaged over 20 runs using random salts for the hash functions.

We also measure the space efficiency of the chained cuckoofilter as compared to a information theoretic optimum. We define the bit efficiency of thefi lter to measure the efficiency as

$$
\text { Efficiency }:=\frac{\text { sketch size in bits }}{n \log _{2} 1 / \rho} \in[1, \infty)
$$

where $n$ is the total number of keys inserted and $\rho$ is the FPR. When all keys are distinct, a sketch with an efficiency of 1 cannot be improved since it matches the information theoretic lower bound given by the denominator.

\subsection{Multiset results}

Figure 3 shows the behavior of a regular multiset cuckoofi lter and cuckoofi lter with chaining as the number of duplicates per key is varied. The chained cuckoofi lter is able to achieve roughly the same load factor regardless of number of duplicates. In contrast, the plain cuckoofi lter's ability to achieve a high load factor quickly decreases. For Zipf-Mandelbrot data, the plain cuckoo hash encounters very few items before it fails. The cuckoofi lter quickly encounters more copies of these keys than it can store. When the number of generated duplicates per key equals the maximum allowed by a non-chained cuckoofilter $d$, the chained and plain cuckoofi lters achieve similar load factors. However, the chained cuckoofi lter has a slightly worse FPR as it inspects an extra bucket pair.

Taking into account both the load factor and the FPR, Figure 8 shows an optimized chained cuckoofi lter obtains a bit efficiency of $\approx 1.93$ for the optimal or nearly optimal setting of $d=3$ when the number of entries per bucket is $b=6$. In comparison, set membership sketches only gain modestly better efficiency for the

\footnotetext{
${ }^{1}$ The Mandelbrot parameters are the parameters from Mandelbrot's original paper [43] which introduced the Zipf-Mandelbrot distribution and provided parameters yielding good empiricalfi ts when modeling word frequencies. [51]
}

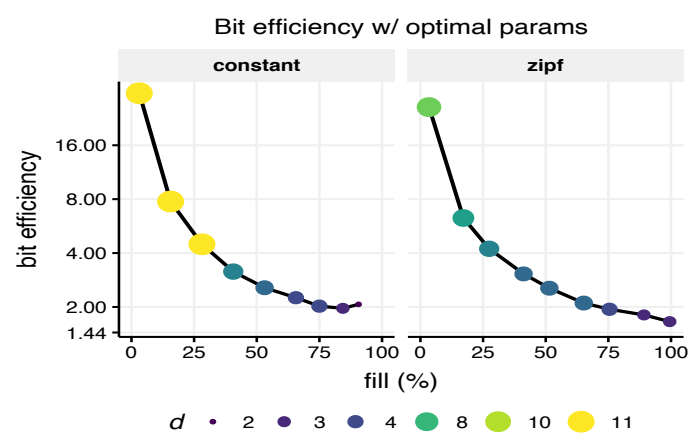

Figure 8: Small values for $d$ duplicates per bucket pair generally use space more efficiently. While a very low value of $d=2$ can achieve higher load factors, it can result in higher FPRs that make the sketch less efficient.

loss in functionality in handling duplicates. A Bloomfi lter has a bit efficiency of $\approx 1 / \log 2 \approx 1.44$. Removing a CCF's chaining functionality to obtain a cuckoofi lter without semi-sorting gives a bit efficiency of $\approx 1.53$ when the FPR $=1 \%$.

\subsection{JOB-light experiment}

Our second set of experiments evaluate the efficacy of CCF's for join processing on a real world dataset. We evaluated the CCF's ability to reduce the output of scan operators using the JOB-light workload [34] derived from the Join Order Benchmark (JOB) [40] used to evaluate the quality of query optimizers.

Given a query and a table in that query, we wish to determine the minimum sized output that a scan operator on that table can provide and compare it to an operator that only applies the predicates on that table. The minimum size output is produced by converting joins of this base table to other tables to semi-joins, which only check if the key exists in the other tables after applying predicates. To execute a semi-join using a CCF, for a row in the base table with key $k$, each joining table's CCF is queried for $(k, \mathcal{P})$ where $\mathcal{P}$ is the predicate from the query for that table. We define the Reduction Factor (RF) to be

$$
\text { Reduction Factor }:=\frac{M_{\text {semijoin }}}{M_{\text {predicate }}}
$$

where $M_{\text {semijoin }}$ is the number of base table rows that both match the given predicates on that table and all other CCF's. The value $M_{\text {predicate }}$ is the number of rows in the base table that match the predicates on that table only. The concept of reduction factor is similar to predicate selectivity: when reduction factor (selectivity) is 0.0 , no rows are selected and when reduction factor (selectivity) is 1.0 , all rows are selected. For a distributed system, the reduction factor measures what proportion of tuples are sent over the network, or in non-distributed hash joins, how much smaller the hash table sizes are. We also measure the CCF size, the space cost of the CCF used to achieve these tuple/size reductions.

8.3.1 JOB-light data and workload. The JOB-light workload consists of 70 queries, each joining up to 5 large tables from the IMDB dataset. Among these 70 queries are 237 instances of tables that qualify for matching join keys and predicates in at least one CCF, effectively a semi-join reducer [5]. 


\begin{tabular}{l|r|l|r} 
Table & $\begin{array}{r}\text { Number } \\
\text { of Rows }\end{array}$ & Predicate & $\begin{array}{r}\text { Column } \\
\text { Cordinality }\end{array}$ \\
\hline cast_info & $36,244,344$ & role_id & 11 \\
movie_companies & $2,609,129$ & company_id & $\mathbf{2 3 4 , 9 9 7}$ \\
movie_companies & $2,609,129$ & company_type_id & 2 \\
movie_info & $14,835,720$ & info_type_id & $\mathbf{7 1}$ \\
movie_info_idx & $1,380,035$ & info_type_id & 5 \\
movie_keyword & $4,523,930$ & keyword_id & $\mathbf{1 3 4 , 1 7 0}$ \\
title & $2,528,312$ & kind_id & 6 \\
title & $2,528,312$ & production_year & $\mathbf{1 3 2}$
\end{tabular}

Table 2: Summary of tables and predicates used in JOB-light workload. High cardinalities in bold.

\begin{tabular}{l|l|l|r|r} 
& & Predicate & Avg & Max \\
Table & Join Key & Column & Dupes & Dupes \\
\hline cast_info & movie_id & role_id & 4.70 & 11 \\
movie_companies & movie_id & company_id & 2.14 & $\mathbf{8 7}$ \\
movie_companies & movie_id & company_type_id & 1.54 & 2 \\
movie_info & movie_id & info_type_id & 4.17 & $\mathbf{6 8}$ \\
movie_info_idx & movie_id & info_type_id & 3.00 & 4 \\
movie_keyword & movie_id & keyword_id & 9.48 & $\mathbf{5 3 9}$ \\
title & id & kind_id & 1.00 & 1 \\
title & id & production_year & 1.00 & 1
\end{tabular}

Table 3: Number of distinct attribute values per key. Equivalently, number of duplicated keys. High Max Dupes in bold.

In this workload, each query involves 2 to 5 of the 6 tables listed in Table 2, and all joins are on the movie identifier. Two tables, movie_companies and title, each contain two predicate columns, thus providing an opportunity to evaluate the workload using a combination of single-attribute and multi-attribute CCF's. While most predicates are equality predicates, 55 JOB-light queries have inequality predicates on title.production_year which ranges from 1880 to 2019. We applied the simple binning technique of Section 7 and mapped the 132 values to 16 roughly equal-sized intervals and converted inequality predicates to in-lists. In the cases where the scan operation was on the title table, we omitted this binning since the predicate could be evaluated directly.

The relevant IMDB data for the JOB-light workload is summarized in Table 2 and Table 3. These include the predicate columns and their cardinalities. When using 4 bit attributefi ngerprints, 4 of the predicates can be considered "high" cardinality with cardinality $>2^{4}$. Table 3 shows the number of duplicate predicate attribute values per join key. This affects both the sizing of the sketches as well as the FPR. While the Avg Dupes vary from 1.00 to 9.48 distinct duplicates per join key, CCF's must handle the worst case behavior of Max Dupes which varies from 1 to 539.

8.3.2 JOB-light experiment setup. We evaluated all four CCF methods: Plain (regular cuckoofi lter allowing duplicate keys); Chained (CCF w/ chaining); Bloom (CCF w/ Bloom); and Mixed (CCF w/ Bloom conversion); as well as a simple cuckoofi lter for a range of attribute sizes,fi ngerprint sizes, and Bloom attribute sketch sizes.

The followingfi lter parameters were evaluated: attributefingerprint sizes of $|\alpha|=4$ or 8 bits,fi ngerprint sizes of $|\kappa|=7,8$, or 12 bits, and Bloomfi lter sizes ranging from 4 to 24 . The number of hash functions used in the Bloomfi lter was eitherfi xed at 2, or was optimized to achieve the lowest FPR under the assumption that 2 attribute vectors are inserted per key. We found the latter setting resulted in uniformly worse FPR's and omit their results from the rest of this analysis. Like the multiset experiment, the maximum number of duplicate keys per bucket pair was always set to $d=3$.

The number of buckets and bucket size were independently chosen for eachfi lter based on the analysis in Section 6.3. Given the predicted number of entries, wefi nd the smallest bucket size which would both result in an acceptable load factor and have high likelihood of successfully inserting all input rows based on the multiset experiment. Note that the predicted number of entries needed can be estimated from the data using a bottom-k [14] or two-level [13] sampling scheme.

\subsection{JOB-light results}

We compare how the CCF methods perform versus the theoretically best possible RF (Reduction Factor), which makes full use of all predicate information, and against the best existing baseline of a Cuckoo Filter, which throws away information about predicates. The best possible RF is the Exact Semijoin RF where no false positives are emitted. We also examined the effect of changing the size of fingerprints and attribute sketches on the accuracy of CCF's.

Largefilters: Figure 9a plots the reduction factor on the y-axis for each of the 237 possible scan operators in the 70 workload queries. The parameters of allfi lters are the same, having 8-bit attributes, 12-bitfi ngerprints, and 4 hash functions for Bloomfilters. Thesefi lters are "large" due to the choice of 8 and 12 bits for attribute andfi ngerprint size respectively, as well as specifying a large Bloomfi lter sketch. The ordering of these tables on the $\mathrm{x}$-axis is in increasing order of the Exact Semijoin reduction factor; therefore, the reduction factor of allfi lters should be to the left and above the Exact Semijoin RF line. For largefi lters, the RF of allfilter methods are fairly close to Exact Semijoin with a few outliers.

Figure 9b also uses largefi lters, but has a different baseline than in 9a. Here the baseline is based on using large Bloomfi lters, but only looking up thefi lters' join keys, ignoring any other predicates. This baseline behavior is analogous to a regular Cuckoofi lter rather than a CCF and represents the current state-of-the-art of pre-built filters. The reduction factor of allfi lters should be to the right and below the Cuckoo Filter baseline. CCF reduction factors are substantially better than current state-of-the-art pre-builtfilters. In many cases, where the Cuckoo Filter reduction factor is 1.0, meaning no reduction at all, the CCF RF's are in the range of $0.05-0.20$.

Smallfilters: Now we consider "small"fi lters, using 4-bit attributes, 7-bitfi ngerprints, and 2 hash functions for Bloom, reducing filter size by more than half. Figures $9 \mathrm{c}$ and $9 \mathrm{~d}$ show the reduction factors by increasing Exact Semijoin and Cuckoo Filter RF baselines respectively, forfi lters of smaller size. Compared to largefilters, the number of non-optimal reduction factors in $9 \mathrm{c}$ are more visible. The separation of Bloom CCF reduction factors from Mixed and Chained is particularly noticeable, while small Mixed and Chained RF's are similar to largefilters. Even small CCF's are substantially better than current state-of-the-artfilters.

Plainfilters: Note that none of thesefi gures have results for Plain CCFfi lters as they did not result in reasonably sizedfilters. The smallest Plainfi lter was larger than every other CCF and had an inefficient load factor of 35\%. Larger attributefi ngerprints result in insertion failures for any reasonablefi lter parameters because 


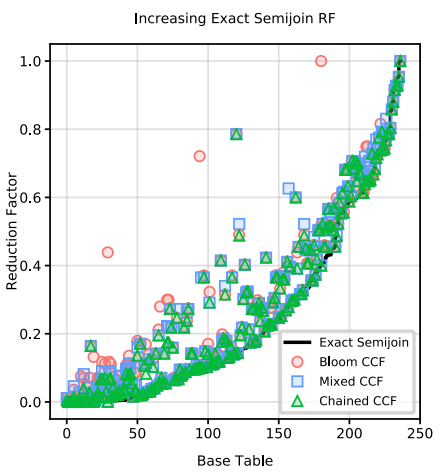

(a) Large vs Exact Semijoin

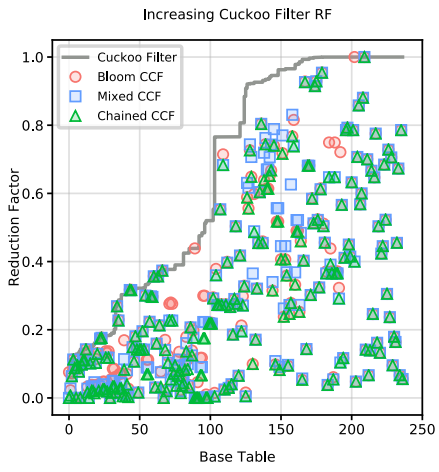

(b) Large vs Cuckoo Filter

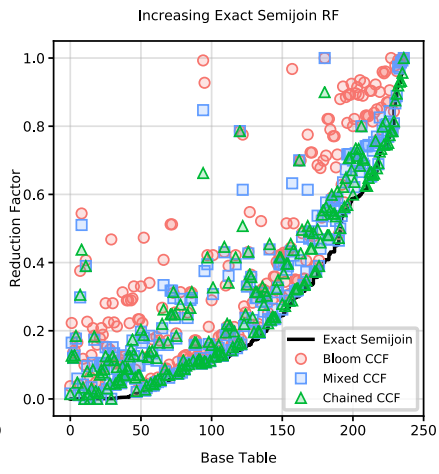

(c) Small vs Exact Semijoin

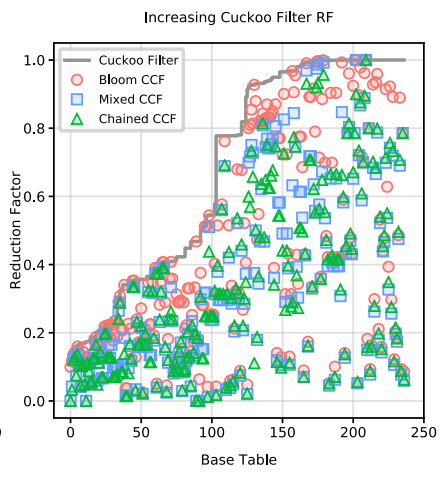

(d) Small vs Cuckoo Filter

Figure 9: Reduction factors for CCF's versus the Exact Semijoin or Cuckoo Filter RF baselines, with largefi lters in 9a, 9b and smallfi lters for 9c, 9d. The reduction factors of CCF's are much improved over Cuckoo Filters and fairly close to the optimal, but impractical, exact semijoin.
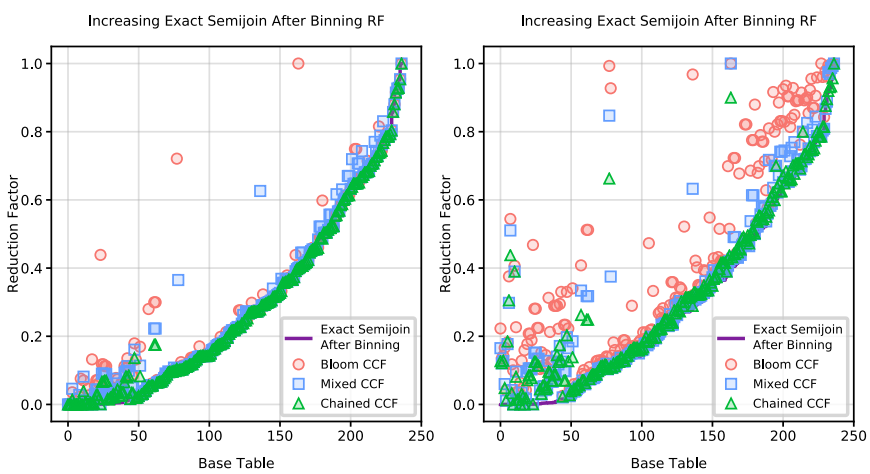

Figure 10: RF's for large (left) and small (right) CCF's versus the exact semijoin baseline after binning production_year. Compared to 9a and 9c, thisfi gure shows the CCF is close to optimal and false positives are mostly due to binning.

the number of distinct attribute values is too large. For example, as shown in Table 3, movie_keyword.keyword_id has 539 distinct duplicates which would require a minimum bucket size of 270 .

8.4.1 JOB-light aggregate results. On aggregate, the reduction factor over all table scans was $\approx 0.28$ using a CCF with chaining and "small" sketches. In contrast, using regular cuckoofi lters with no predicate information resulted in a reduction factor of $\approx 0.68$. The best possible reduction factor obtained from performing an exact semi-join was 0.20 . Furthermore, half of the difference in reduction factors between the CCF and exact semi-join is explained by the binning on the range predictate on production_year as seen in figures 10 and 11. If an exact semi-join is performed on data with binned production_year, the optimal reduction factor is 0.24 .

Furthermore, using the largest sized CCF, which was a CCF with chaining, 12 bit keyfi ngerprints, and 8 bit attributefingerprints, the FPR was just $0.8 \%$ relative to a semi-join with binned production_year, and the reduction factor was 0.245 . The FPR including errors due to binning was $6.1 \%$.

Figure 12 shows that the benefits of CCF's are compounded as more joins are added. Figure 13 shows the size of a CCF relative to

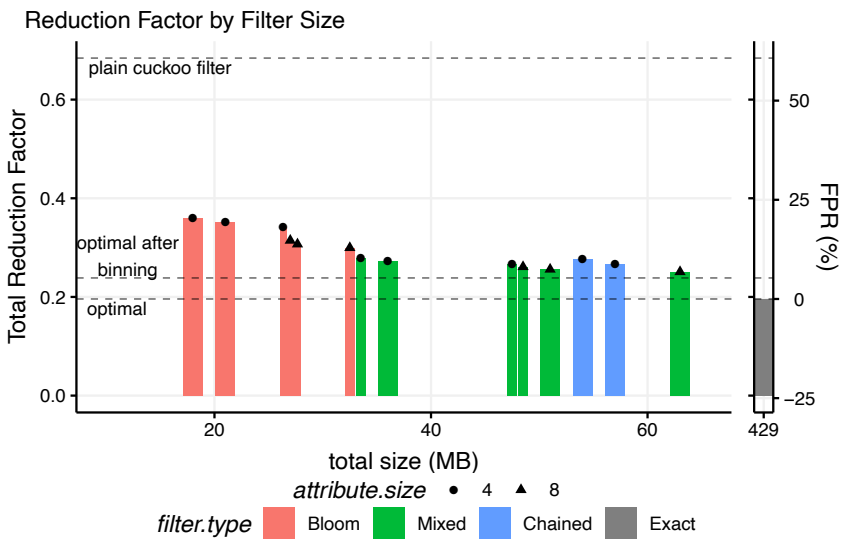

Figure 11: Overall RF and FPR byfi lter type and size. CCF's obtain better RF while using an order of magnitude less space than a hash table performing exact membership testing. Bloom attribute sketches resulted in the smallest sizes. Mixed attribute sketches using Bloom conversion achieved the smallest FPR for a given amount of space.

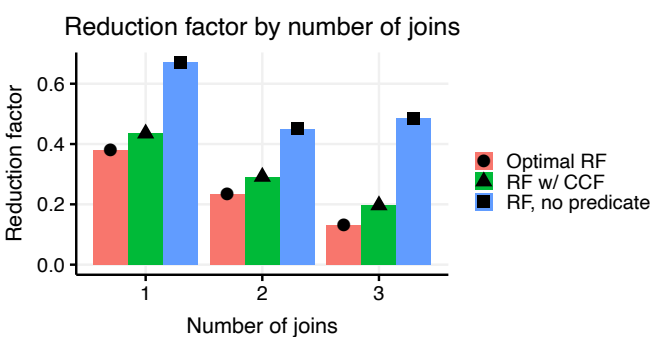

Figure 12: Using CCF's with predicates results in multiplicative benefits in reducing output sizes.

the raw data. Each CCF represents a movie id and the given predicate column. The gains from each CCF can varying significantly based on the underlying data and the number of duplicate keys. 


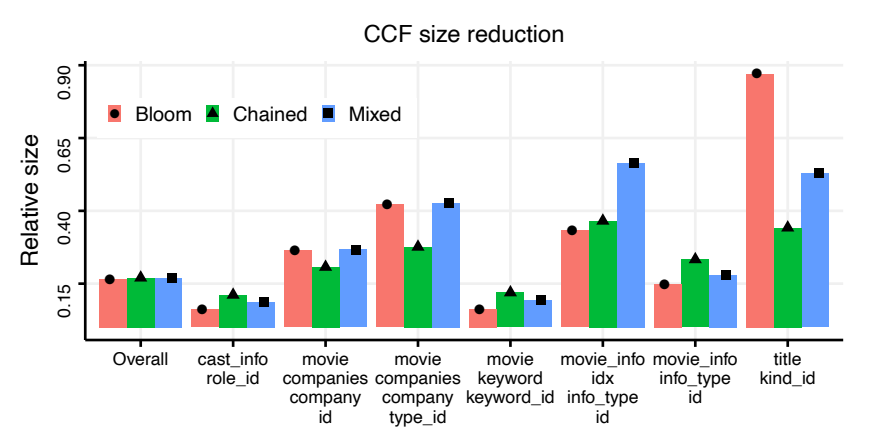

Figure 13: Each CCF's size strongly depends on the underlying data. Bloomfi lters yield larger reductions for tables with many duplicated keys while chaining yields larger reductions on those with unique keys.

8.4.2 CCF size results. We did notfi nd that one method clearly outperformed others at any given size. However, only Bloomfilter attribute sketches could produce very small sketches. The smallest set of sketches required 18.5 megabytes. By comparison, if keys and high cardinality attributes are stored using 32 bits and low cardinality attributes are stored with 8 bits, then the raw data used in the sketches requires 322 megabytes of space. A open addressing hash table would require 429 megabytes to store these if it could achieve a $75 \%$ load factor. The much smaller sizes of the CCF are due both to sketching keys and attributes as well as due to elimination of duplicate keys.

Figure 11 shows the reduction factor as a function of the total size of all sketches for various parameter settings. The maximum sketch size was restricted to 60 megabytes. Bloomfi lter attribute sketches yield the smallest possible sketches since they store no duplicates, but they also yield the highest FPR of up to $20.4 \%$. The mixed attribute sketches which use attributefi ngerprint vectors and switch to Bloomfi lters when there are too many duplicates were able to retain most of the benefits of attributefingerprint vectors with significantly less space.

For parameters we considered, given two sketches with the same size, allocating more space to the attribute sketch yielded a smaller FPR than allocating more space to the keyfi ngerprint size. This can be seen in Figure 11 in cases where there are adjacent, identically colored half-width bars but different marks on the bar.

Figure 11 shows the space versus reduction factor tradeofffor different $\mathrm{CCF}$ types. At 35 megabytes, the reduction factor of a CCF with mixed attributed sketches is within $10 \%$ of the optimal and within $5 \%$ of the best a CCF can do with binned production_year. While larger CCF's further improve the FPR, the practical benefit in the reduction factor is minimal. A 35 megabyte set of CCF's represents an over $10 \times$ reduction in size in memory. If string based columns are included, which are very common in real world databases [59], we expect the space savings to be much greater.

\subsection{Implementation and run-time performance}

We implemented a single threaded $\mathrm{CCF}$ in $\mathrm{C}++$ and ran our experiments using a single Intel(R) Xeon(R) CPU E5-2630 v4 @ 2.20GHz core running CentOS Linux 7. We used the Jenkins lookup3 hash function [29] used by the original cuckoofi lter paper [20]. Allfilter methods could process 1 million matches per second.

\section{DISCUSSION AND FUTURE WORK}

While we focus on the application of CCF's to join processing, the sketch itself can be seen as a sketch of the entire input table with a hash based index on the key. Furthermore, the chaining technique can also be used to allow regular cuckoo hash tables, which store the full key, to store duplicates. Thus, we believe the sketch and its methods have more general applications beyond join processing.

For example, recent work on Cuckoo Indexes [32] enables data skipping for secondary columns by associating a key for the column to the multiple data partitions containing it. The CCF provides a simple, new solution to the resulting multiset problem.

Another possible application is for access control in information retrieval. Documents in a database may be restricted by subscription status, country, or other factors. However, a typical search engine using inverted indexes may not be able to efficiently restrict results to documents a user has access to. A CCF on document, permission pairs can help efficientlyfi lter the search results. Another possible use is for hierarchical set membership queries. For example, a database may store documents with revision numbers, modification times, or other traits across a collection of servers. A CCF could be used to check if a cache contains a specific document revision, and if not, whether there is any revision already in the cache. It can further aid in resource routing.

Although the sketch is made robust to skew in the key distribution, sizing the sketch is still problematic as it requires predicting the number offi lled entries in thefi lter. Future work to improve this method, as well as many other ASM sketches, includes enabling dynamic adjustment of the size of the sketch.

Furthermore, while we present empirical evidence that on data containing duplicate keys, the CCF with chaining can achieve a load factor comparable to that of a regular cuckoofi lter acting on data with no duplicates, we do not have a theoretical proof that this is always the case.

\section{CONCLUSIONS}

We introduce conditional cuckoofi lters, a new sketch for approximate set membership queries which enables equality predicates to be added to queries. This yields at least two significant advantages in join processing. First, it enablesfi lters that are specific to the predicate to be applied to both build and probe sides of a join, not just the probe side. This increases the number of cases where the data structures created on the build sidefi ts into main memory. Second, it enables predicate pushdown from one table to all other tables in the transitive closure of the join graph.

We propose, analyze, and evaluate multiple variations of $\mathrm{CCF}$ sketches. In particular, we extend cuckoo hash tables using a chaining technique that makes it robust to duplicated keys and allows high load factors to be achieved. All variations reduced the number of rows emitted by a scan operator to close to the optimal number on the workload and did so with substantial space savings. This represents a significant improvement over existingfi lters that do not support predicates. The properties of the sketches are analyzed which allows practitioners to predict the performance of the sketches and to choose appropriate parameters for them. 


\section{REFERENCES}

[1] K. Alexiou, D. Kossmann, and P.-Å. Larson. Adaptive rangefi lters for cold data: Avoiding trips to siberia. Proceedings of the VLDB Endowment, 6(14):1714-1725, 2013

[2] Apache. Cassandra. http://cassandra.apache.org/.

[3] Apache. Hbase. http://hbase.apache.org/.

[4] M. A. Bender, M. Farach-Colton, R. Johnson, R. Kraner, B. C. Kuszmaul, D. Med jedovic, P. Montes, P. Shetty, R. P. Spillane, and E. Zadok. Don't thrash: how to cache your hash onflash. Proceedings of the VLDB Endowment, 5(11):1627-1637, 2012.

[5] P. A. Bernstein, P. A. Bernstein, N. Goodman, E. Wong, C. L. Reeve, and J. B. Rothnie Jr. Query processing in a system for distributed databases (sdd-1). ACM Transactions on Database Systems (TODS), 6(4):602-625, 1981.

[6] B. H. Bloom. Space/time trade-offs in hash coding with allowable errors. Communications of the ACM, 13(7):422-426, 1970

[7] F. Bonomi, M. Mitzenmacher, R. Panigrahy, S. Singh, and G. Varghese. An improved construction for counting bloomfi lters. In European Symposium on Algorithms, pages 684-695. Springer, 2006.

[8] K. Bratbergsengen. Hashing hethods and relational algebra operations. In Proceedings of the 1984 Very Large Database Conference. Citeseer, 1984.

[9] A. D. Breslow and N. S. Jayasena. Mortonfi lters: Faster, space-efficient cuckoo filters via biasing, compression, and decoupled logical sparsity. Proc. VLDB Endow., 11(9):1041-1055, May 2018.

[10] A. Broder and M. Mitzenmacher. Network applications of bloomfi lters: A survey. Internet mathematics, 1(4):485-509, 2004.

[11] A. Broder, M. Mitzenmacher, and A. B. I. M. Mitzenmacher. Network applications of bloomfi lters: A survey. In Internet Mathematics, pages 636-646, 2002

[12] P. Brown and P. Haas. Bhunt: Automatic discovery of fuzzy algebraic constraints in relational data. In $V L D B, 2003$

[13] Y. Chen and K. Yi. Two-level sampling for join size estimation. In SIGMOD. ACM, 2017

[14] E. Cohen and H. Kaplan. Summarizing data using bottom-k sketches. In PODC, 2007

[15] D. Das, J. Yan, M. Zait, S. R. Valluri, N. Vyas, R. Krishnamachari, P. Gaharwar, J. Kamp, and N. Mukherjee. Query optimization in oracle 12c database in-memory. Proc. VLDB Endow., 8(12):1770-1781, Aug. 2015.

[16] M. Eich, P. Fender, and G. Moerkotte. Faster plan generation through consideration of functional dependencies and keys. Proc. VLDB Endow., 9:756-767, 2016.

[17] M. Elhemali, C. A. Galindo-Legaria, T. Grabs, and M. M. Joshi. Execution strategies for sql subqueries. In Proceedings of the 2007 ACM SIGMOD international conference on Management of data, pages 993-1004, 2007.

[18] D. Eppstein. Cuckoofi lter: Simplification and analysis. In 15th Scandinavian Symposium and Workshops on Algorithm Theory (SWAT 2016). Schloss DagstuhlLeibniz-Zentrum fuer Informatik, 2016.

[19] Facebook. Rocksdb. https://github.com/facebook/rocksdb.

[20] B. Fan, D. G. Andersen, M. Kaminsky, and M. D. Mitzenmacher. Cuckoofilter: Practically better than bloom. In Proceedings of the 10th ACM International on Conference on emerging Networking Experiments and Technologies, pages 75-88. ACM, 2014.

[21] L. Fan, P. Cao, J. Almeida, and A. Z. Broder. Summary cache: A scalable wide-area web cache sharing protocol. In ACM SIGCOMM Computer Communication Review volume 28, pages 254-265. ACM, 1998.

[22] C. A. Galindo-Legaria, T. Grabs, S. Gukal, S. Herbert, A. Surna, S. Wang, W. Yu P. Zabback, and S. Zhang. Optimizing star join queries for data warehousing in microsoft sql server. In ICDE, pages 1190-1199. IEEE, 2008.

[23] Google. Leveldb. https://github.com/google/leveldb/.

[24] T. M. Graf and D. Lemire. Xorfi lters: Faster and smaller than bloom and cuckoo filters. arXiv preprint arXiv:1912.08258, 2019.

[25] P. G. D. Group. Postgresql. https://www.postgresql.org/.

[26] P. Haas, I. F. Ilyas, G. Lohman, and V. Markl. Discovering and exploiting statistical properties for query optimization in relational databases: A survey. Stat. Anal. Data Min., 1:223-250, 2009.

[27] B. Hentschel, M. S. Kester, and S. Idreos. Column sketches: A scan accelerator for rapid and robust predicate evaluation. SIGMOD, 2018

[28] I. F. Ilyas, V. Markl, P. Haas, P. Brown, and A. Aboulnaga. Cords: automatic discovery of correlations and soft functional dependencies. In SIGMOD '04, 2004

[29] B. Jenkins. Jenkins lookup3 hash function. http://www.burtleburtle.net/bob/c/ lookup3.c.

[30] S. Kandula, L. Orr, and S. Chaudhuri. Pushing data-induced predicates through joins in big-data clusters. Proc. VLDB Endow., 13(3):252-265, Nov. 2019.

[31] H. Kimura, G. Huo, A. Rasin, S. Madden, and S. Zdonik. Correlation maps: A compressed access method for exploiting soft functional dependencies. Proc. VLDB Endow., 2:1222-1233, 2009.

[32] A. Kipf, D. Chromejko, A. Hall, P. Boncz, and D. G. Andersen. Cuckoo index: a lightweight secondary index structure. Proc. VLDB Endow., 13(13):3559-3572, 2020.
[33] A. Kipf, T. Kipf, B. Radke, V. Leis, P. Boncz, and A. Kemper. Learned cardinalities: Estimating correlated joins with deep learning. CIDR, 2019.

[34] A. Kipf, T. Kipf, B. Radke, V. Leis, P. A. Boncz, and A. Kemper. Learned cardinalities: Estimating correlated joins with deep learning. ArXiv, abs/1809.00677, 2018.

[35] M. Kitsuregawa, H. Tanaka, and T. Moto-Oka. Application of hash to data base machine and its architecture. New Generation Computing, 1(1):63-74, 1983.

[36] M. Kornacker, A. Behm, V. Bittorf, T. Bobrovytsky, C. Ching, A. Choi, J. Erickson, M. Grund, D. Hecht, M. Jacobs, et al. Impala: A modern, open-source sql engine for hadoop. In CIDR, 2015

[37] T. Lahiri, S. Chavan, M. Colgan, D. Das, A. Ganesh, M. Gleeson, S. Hase, A. Holloway, J. Kamp, T.-H. Lee, et al. Oracle database in-memory: A dual format in-memory database. In 2015 IEEE 31st International Conference on Data Engineering, pages 1253-1258. IEEE, 2015

[38] H. Lang, T. Neumann, A. Kemper, and P. Boncz. Performance-optimalfiltering: Bloom overtakes cuckoo at high throughput. Proceedings of the VLDB Endowment, 12(5):502-515, 2019

[39] T. Lee, K. Kim, and H.-J. Kim. Join processing using bloomfi lter in mapreduce. In Proceedings of the 2012 ACM Research in Applied Computation Symposium, RACS '12, pages 100-105, New York, NY, USA, 2012. ACM.

[40] V. Leis, A. Gubichev, A. Mirchev, P. Boncz, A. Kemper, and T. Neumann. How good are query optimizers, really? Proceedings of the VLDB Endowment, 9(3):204-215, 2015.

[41] C. Luo and M. J. Carey. Lsm-based storage techniques: a survey. The VLDB fournal, 29(1):393-418, 2020

[42] L. F. Mackert and G. M. Lohman. $R^{*}$ optimizer validation and performance evaluation for distributed queries. In Proceedings of the 12th International Conference on Very Large Data Bases, VLDB '86, pages 149-159, San Francisco, CA, USA, 1986. Morgan Kaufmann Publishers Inc.

[43] B. Mandelbrot. An informational theory of the statistical structure of language. Communication theory, 84:486-502, 1953.

[44] M. Mitzenmacher. Compressed bloomfilters. IEEE/ACM Transactions on Networking (TON), 10(5):604-612, 2002.

[45] J. K. Mullin. Optimal semijoins for distributed database systems. IEEE Trans. Softw. Eng., 16(5):558-560, May 1990.

[46] J. K. Mullin. Estimating the size of a relational join. Information Systems, 18(3):189 - 196, 1993.

[47] Oracle. Using oracle database $11 \mathrm{~g}$ release 2 result cache in an oracle rac environment. https://www.oracle.com/technical-resources/articles/database/ datawarehouse-vallath-resultcache-rac.html.

[48] A. Pagh, R. Pagh, and S. S. Rao. An optimal bloomfi lter replacement. In Symposium on Discrete Algorithms, pages 823-829. Society for Industrial and Applied Mathematics, 2005

[49] R. Pagh and F. F. Rodler. Cuckoo hashing. fournal of Algorithms, 51(2):122-144, 2004.

[50] P. Pandey, M. A. Bender, R. Johnson, and R. Patro. A general-purpose counting filter: Making every bit count. SIGMOD, 2017.

[51] S. T. Piantadosi. Zipf's word frequency law in natural language: A critical review and future directions. Psychonomic bulletin \& review, 21(5):1112-1130, 2014.

[52] F. Putze, P. Sanders, and J. Singler. Cache-, hash-and space-efficient bloom filters. In International Workshop on Experimental and Efficient Algorithms, pages 108-121. Springer, 2007.

[53] D. L. Quoc, I. E. Akkus, P. Bhatotia, S. Blanas, R. Chen, C. Fetzer, and T. Strufe. Approxjoin: Approximate distributed joins. In Proceedings of the ACM Symposium on Cloud Computing, SoCC '18, pages 426-438, New York, NY, USA, 2018. ACM.

[54] SAP. Adaptive server enterprise $15.7 \mathrm{sp} 100$ - new features guide.

[55] R. Sears and R. Ramakrishnan. blsm: a general purpose log structured merge tree. In SIGMOD, pages 217-228. ACM, 2012

[56] P. Seshadri, J. M. Hellerstein, H. Pirahesh, T. C. Leung, R. Ramakrishnan, D. Srivastava, P. J. Stuckey, and S. Sudarshan. Cost-based optimization for magic: Algebra and implementation. 1996.

[57] P. Seshadri, H. Pirahesh, and T. C. Leung. Complex query decorrelation. In Proceedings of the Twelfth International Conference on Data Engineering, pages 450-458. IEEE, 1996.

[58] S. Tarkoma, C. E. Rothenberg, and E. Lagerspetz. Theory and practice of bloomfilters for distributed systems. IEEE Communications Surveys \& Tutorials, 14(1):131$155,2011$.

[59] A. Vogelsgesang, M. Haubenschild, J. Finis, A. Kemper, V. Leis, T. Muehlbauer, T. Neumann, and M. Then. Get real: How benchmarks fail to represent the real world. In Workshop on Testing Database Systems, 2018.

[60] M. Wang, M. Zhou, S. Shi, and C. Qian. Vacuumfi lters: more space-efficient and faster replacement for bloom and cuckoofilters. Proceedings of the VLDB Endowment, 13(2):197-210, 2019.

[61] A. Weininger. Efficient execution of joins in a star schema. In $S I G M O D$, pages 542-545. ACM, 2002.

[62] M. Ziauddin, A. Witkowski, Y. J. Kim, J. Lahorani, D. Potapov, and M. Krishna. Dimensions based data clustering and zone maps. Proc. VLDB Endow., 10:16221633, 2017. 